International Journal of Social Research
(ISSN:2576-5531)

\title{
Identity and Political Media Literacy of Youths in Hong Kong
}

\section{Lei Zhang}

School of Journalism and Communication, Jinan University, Guangzhou 510632, China

\section{ABSTRACT}

Since Hong Kong returned to China in 1997, it's local politics and economy have greatly developed. However, in recent years, political turmoil in Hong Kong often occurs, and youth groups have played a radical role in it. Facing various contradictions and conflicts in such a complicated political environment, correctly understanding political messages conveyed by the media, establishing positive attitude in political participation, and scientifically and rationally engaging in political participation activities, not only help Hongkong's youths healthy growth and contribute to the stable development of Hong Kong society. Using a quota sampling method, the Social Investigation and Research Center of Jinan University conducted a questionnaire survey from November 2016 to January 2017.The study found that Hong Kong's youths pay more attention to the identity of "Hong Kong people", pay less attention to political topics, and are less willing to participate in political action. The identity of Hong Kong youth and political media literacy will directly affect the political orientation of Hong Kong society.

Keywords: Hong Kong youth Identity media Political literacy

The paper is supported by National Social Science Foundation of China (13CSH077)
*Correspondence to Author:

Lei Zhang

School of Journalism and Communication, Jinan University

Guangzhou 510632, China

How to cite this article:

Lei Zhang.Identity and Political Media Literacy of Youths in Hong Kong. International Journal of Social Research, 2018; 2:24.

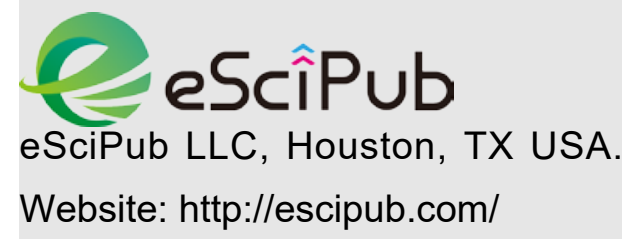




\section{Introduction}

In recent years, there have been conflicts between Hong Kong and the Chinese mainland, and political turmoil has caused a strong reaction from the public. Some Hong Kong youths who participated in political activities have gradually grown into a political core force. The political system in Hong Kong is different from that in mainland China, and the media environment is also different. Citizens pursue democracy and freedom in the use and participation of the media. Some radical Hong Kong youths have a high enthusiasm for political participation. While promoting social democracy, they may also hinder the development of democracy. The political factions in Hong Kong are complex, and some extreme opinions will spread wildly in the Internet. If there is a lack of good political media literacy, teenagers can easily be confused by rumors and make inappropriate comments. It deserves a special attention that the attitudes of Hong Kong youths in choosing and understanding the views of various political factions and ways of expressing their own political comments. Their identity and political media literacy are closely related to Hong Kong's future social stability.

\section{Research methods}

\subsection{Object of study}

This study is based on Hong Kong youths. The age definition of youths is quite different in different areas of research. In the Modern Chinese Dictionary, the definition of youths is the stage of life between 15,16 to 30 years old 1 . In the Wikipedia Chinese version, youths is defined as a specific age group from 19 to 39 years old ${ }^{2}$. The age range of young people is 14 to 35 years old in the official Chinese statistics ${ }^{3}$. In Hong Kong, the Hong Kong Federation of Youth Groups, established in 1960, stipulates that "Hong Kong residents aged 6-35 years old may apply to become members of the Youth Association"4. The "Top Ten Outstanding Youth Elections" established by Junior Chamber International of Hong Kong set the selection criteria as "Hong Kong youths between the ages of 21 and 40"5. Youth in the World Youth Report published by the United Nations is defined as a population between the ages of 15 and $24^{6}$. Although the UN's UNESCO has followed the above criteria in international activities, it also believes that when conducting youth activities, different youth definitions should be used according to specific situations ${ }^{7}$. Furthermore the UNESCO emphasize that youth is an evolving mixed group, "young" as an experience varies greatly from region to region, and even within the country8.

There are a lot of different definitions of the age of young people. In order to adapt to the actual population development in Hong Kong, this study adopts a more loose scale, with 14 to 45 years old as the definition of youths.

Affected by historical and practical factors, Hong Kong's media environment is relatively free. Growing up in such an environment, Hong Kong

\footnotetext{
[2017-01-20].

5 Junior Chamber International of Hong Kong: http://www.jcihk.org/toyp/chief/.[2017-01-20].

6 United Nations: <World Youth

Report >http://www.unworldyouthreport.org/images/docs/un_world_y outh_report_youth_civic_engagement.pdf.

$78^{\text {th }}$ UNESCO Youth Forum: http://www.unesco.org/new/zh/youthforum-2013/.[2017-01-20].

8 UNESCO: <About the Youth Program>

http://www.unesco.org/new/en/social-and-human-

sciences/themes/youth/about-youth/.
} 
youths have gradually entered the stage of the political movement and become an influential political force. It is of great significance for understanding the true public opinion of Hong Kong society to study that Hong Kong youths actually obtained political information and to analyze their attitudes and behaviors towards political participation.

\subsection{Methods of study}

In order to ensure the representativeness of the sample and the realistic operability of the sampling, the sampling plan adopts the method of quota sampling. According to the official demographic data published by the Statistics Department of the Hong Kong SAR Government, the sampling quota ratio is designed based on gender, age and region as the basic elements. The study used five trained graduate students and 60 trained undergraduates (including 43 Hong Kong students) as investigators to go to Hong Kong for a field survey. The survey area covers Hong Kong's land administrative divisions, including Hong Kong Island, Kowloon and the New Territories. (Since the New Territories has a large population and a large area, it is subdivided into New Territories East and New Territories West.) In order to meet the actual situation of Hong Kong's local culture, the survey questionnaire was designed in traditional Chinese characters.

The implementation time of the sample survey was from November 2016 to January 2017. A total of 1291 questionnaires were issued. After removing the questionnaires that were incomplete and unqualified and did not meet the sample specifications, 1227 valid questionnaires were collected, and the effective recovery rate was $95.0 \%$. After screening, correcting and coding all the questionnaires, the data was entered through Epidata software, and finally imported into the statistical analysis software SPSS22.0 to analyze and process the data. In addition to conducting a questionnaire survey of young people in the target age group in Hong Kong, 25 Hong Kong youths aged between 14 and 45 were also deeply interviewed as a supplement to the survey sample.

\section{Sample of study}

In terms of gender ratio, male and female respondents in the survey sample accounted for $43.4 \%$ and $56.6 \%$ of the total, respectively. The average age of the sample was 28.9 years and the standard deviation was 9.02. Among the respondents, $39.8 \%$ were age of $14-25,32.7 \%$ were age of $26 \sim 35$, and $27.5 \%$ were $36 \sim 45$. In terms of education level, $6.6 \%$ of the respondents were educated in junior high school or below, and $33.1 \%$ of the respondents had high school or preparatory courses (that is, college preparatory classes in the old school system). And $18.2 \%$ and $7.9 \%$ of the respondents respectively had post-secondary non-degree and associate degree, and $34.1 \%$ of the respondents with bachelor degree or above; From the perspective of occupation, the largest number of respondents were students, reaching $26.3 \%$, followed by clerks, accounting for $12.6 \%$; manager/administrators accounting for $12.0 \%$; and $11.8 \%$ of respondents were engaged in service industries.

In the case of the specific party participation of the respondents, $92.5 \%$ of the respondents indicated that they did not participate in any party. Only $7.4 \%$ of the respondents indicated that they had their own party, among which the majority of the sample participated in the pan-democratic group, accounting for $2.3 \%$; the local selfdetermination group accounted for $1.7 \%$; The moderate groups accounted for $1.6 \%$; The proestablishment camp accounted for $1.0 \%$; the 
rest $0.8 \%$ of the people did not disclose their party.

\section{Hong Kong youth identity}

Identity is a key issue in the field of interdisciplinary cultural studies. Stuart Hall believes that there are two different ways of thinking about "cultural identity", one is that "cultural identity" is a collective, shared culture that is hidden in superficial or artificially imposed "self", and people who share this "self" share a history and ancestor; the other can also be called a constructivist stand which believes that cultural identity is not an eternal, fixed and essential past, and is "existent", "moving" and "changing", and it is a "playing" that constantly succumbs to power, history and culture(Fu \& Zhang, 2016; Hall \& Gay, 2010; Luo \& Liu, 2000). The identity mentioned in this study refers to a person's recognition and acceptance of the role and characteristics of the group, is a collective, shared cultural identity (Deaux, 1993).

\subsection{More Hong Kong youths agreed with the identity of Hong Kong people}

Hong Kong youths agreed with the identity of Hong Kong people. In terms of identity, $43.4 \%$ of respondents considered themselves to be "Hong Kong people", and $30.2 \%$ of respondents considered themselves to be "Hong Kong people in China", accounting for $73.6 \%$ of the total.

In addition, $10.3 \%$ and $9.9 \%$ of the respondents chose "Chinese" and "Chinese in Hong Kong" respectively, and only $4.7 \%$ of respondents had difficulty in explaining their identity. It can be seen that in terms of personal identity, most of the respondents agreed with their identity as "Hong Kong people". The national identity of "Chinese" was obviously weak compared with the local identity of "Hong Kong people".

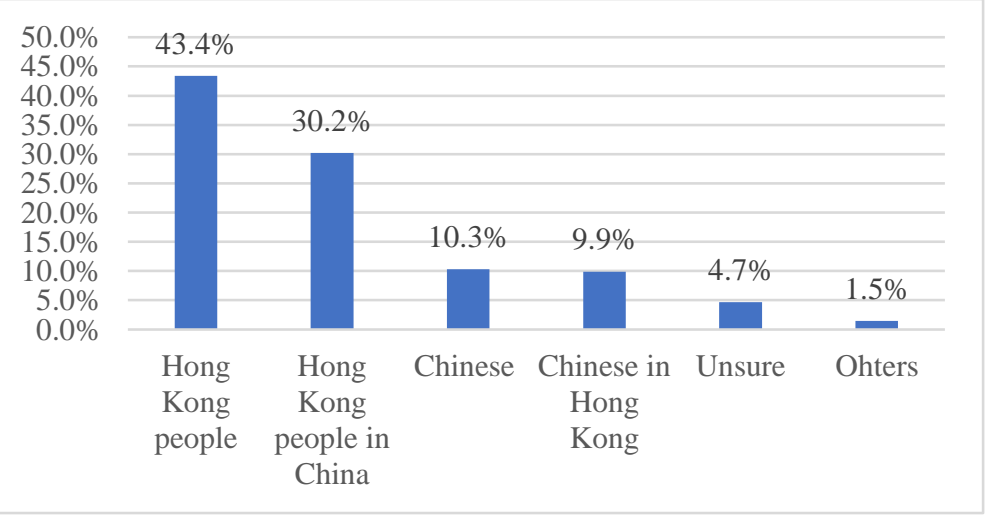

Figure 1: Identity trends $(\mathrm{N}=1197)$

4.2 Hong Kong youths had higher the current living conditions, and only $16.3 \%$ satisfaction with their current lives were considered to be dissatisfied and very

The respondents had a positive attitude towards dissatisfied. Taking the intermediate value 3 of the evaluation of living conditions. A total of $38.5 \%$ the 5 -level scale as the test value, the singleof the respondents indicated that they are very satisfied and satisfied with the current life. $45.2 \%$ sample $T$ test find that the average satisfaction of the respondents were generally satisfied with of the respondents to current life is 3.25 , which is significantly higher than the median value of 3 
$(t=10.425, P<0.001)$.

Life satisfaction is closely related to the political and economic conditions of the region. Hong Kong's economy is developed and the political environment is relatively liberal and democratic.
After returning to China, the overall development level has been rapidly improved. The high level of life satisfaction indicates that respondents have higher recognition of Hong Kong's development.

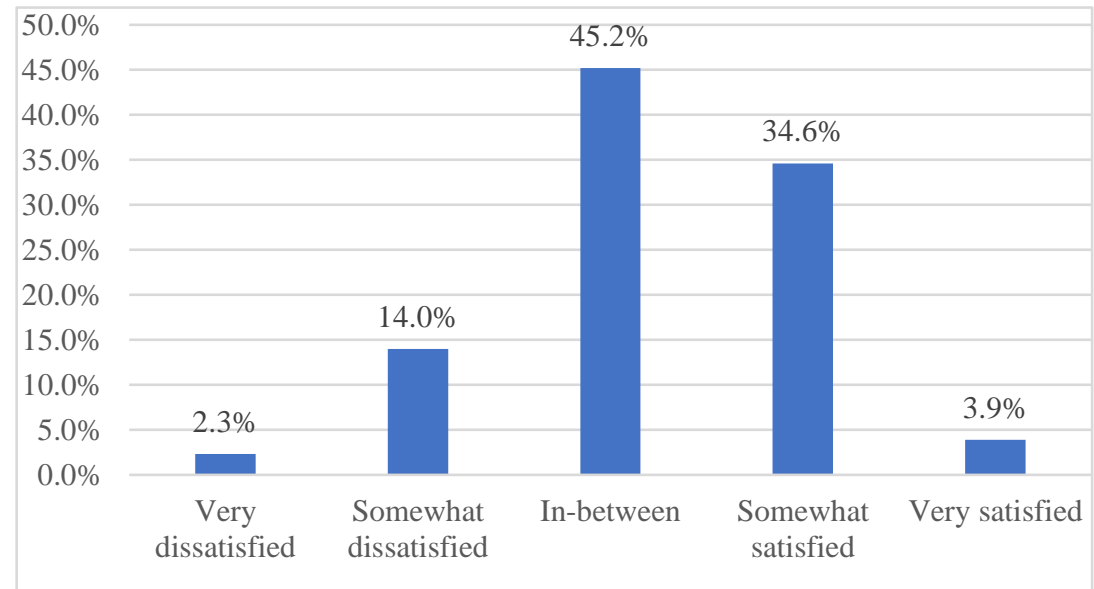

Figure 2: Life satisfaction assessment $(\mathrm{N}=1195)$

\subsection{Hong Kong youths had confidence in the future development of individuals}

When asked if respondents have confidence in their future life, $4.6 \%$ of respondents said that they are very confident about their future development, $26.9 \%$ are more confident, $53.8 \%$ are generally confident, and only $14.8 \%$ of respondents indicated no confidence in future development.

The confidence value of Hong Kong youth interviewed for future life development is 3.19 , which is significantly higher than the median value of $3(t=8.552, P<0.001)$. This shows that the Hong Kong youth interviewed are more confident about their future development and have a positive attitude.

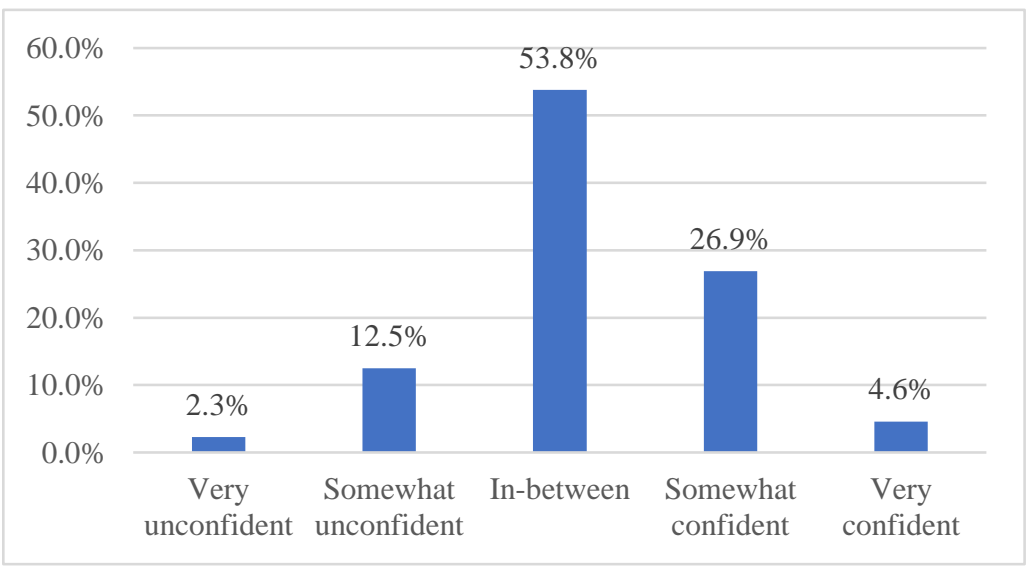

Figure 3: Personal confidence in future life development $(\mathrm{N}=1198)$ 
4.4 Hong Kong youth were optimistic about the future development of Hong Kong

When investigating whether respondents are optimistic about the future development of Hong Kong society, this study used a rating of 1 to 10 points, 1 point represents extreme pessimism, 10 points represents extreme optimism, and the transition of 1 to 10 points represents that extreme negative gradually turns to be extremely positive. It shows that $25.3 \%$ of respondents chose 5 points, $18.8 \%$ of respondents chose 6 points, $23.5 \%$ of respondents scored more than 7 points, and only $2.9 \%$ of respondents scored 1 point. It shows that most of the respondents were more positive and optimistic about the future development of Hong Kong society.

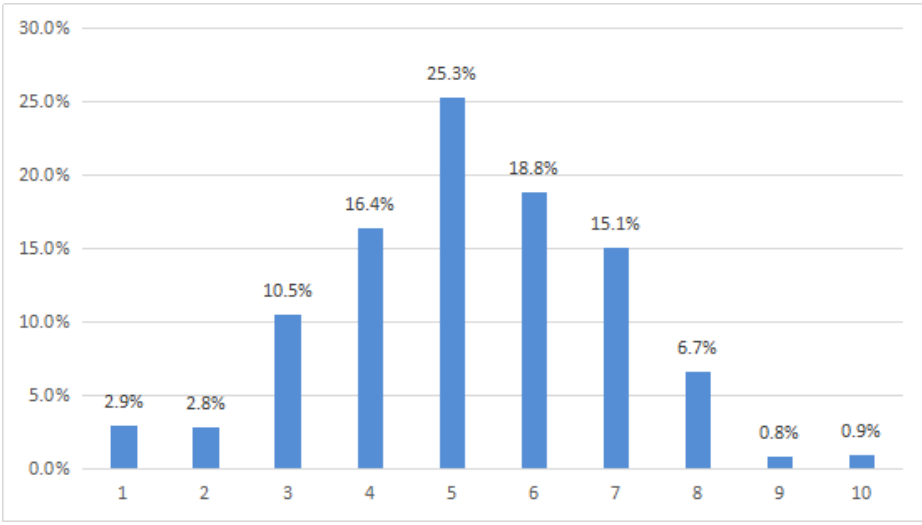

Figure 4: Optimism about the future development of Hong Kong society $(N=1168)$

In summary, according to the above statistical analysis, it is clear that the respondents were satisfied with their current life, and they could take a more positive view of their future development. At the same time, they were generally optimistic about the future of Hong Kong society.

\section{Media political literacy of Hong Kong youths}

5.1 Hong Kong youth were not interested in political news

$44.7 \%$ of Hong Kong respondents were less interested in political issues. Respondents who were not interested in political issues accounted for $30.3 \%$ of the total, and only $25.0 \%$ of respondents showed some interest in political issues. The one-sample $\mathrm{T}$ test shows that the average political interest of the young people interviewed in Hong Kong was 2.90, which was significantly lower than the median of $3(t=-3.530$, $P<0.001)$.

At the same time, in face-to-face interviews, respondents also expressed low attention to political news and were more willing to watch economic news.

"At present, many Hong Kong people do not have a natural and strong sense of political participation. It is simply because there is too many political news in the society, which leads many people to think that we all have strong political participation. Compared with political development, I prefer to pay more attention to economic development." (Female 29-year-old free entrepreneur) 


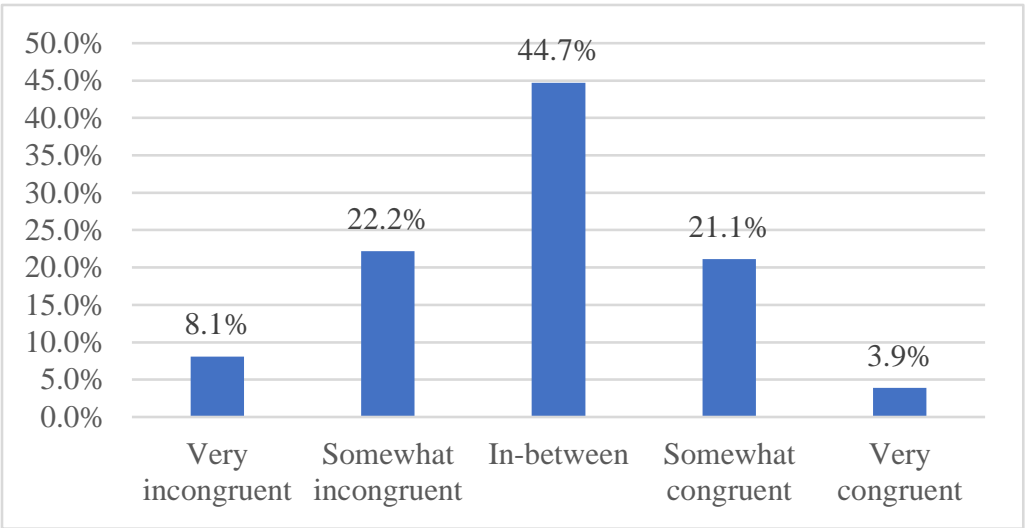

Figure 5: "I am very interested in political issues" ( $N=1196)$

\subsection{Hong Kong youths lacked the enthusiasm for discussing political topics}

There have been many young people involved in the radical political events, and many negative public opinions have been created in Hong Kong in recent years. However, for most ordinary youths living in Hong Kong, were not interested in political topics. In the survey, only $16.2 \%$ of Hong Kong youths were willing to disclose their political opinions and comments. They generally lacked the enthusiasm to discuss political topics. The one-sample $T$ test shows that the average value of this option was 2.63, which was significantly lower than the median value $(t=-$ 13.30, $\mathrm{P}<0.01)$.

This shows that in the overall trend, Hong Kong youths had shown a very cautious attitude towards publicizing their own political opinions and comments. In comparison, the lower the level of education and the older the age, the less likely people were to express their political opinions. In an in-depth interview, a 45-year-old interviewee said frankly:

"I usually discuss political topics, but relatively speaking, I don't often disclose my own political opinions, because when people disagree, it is easy to cause conflicts. I rarely use Twitter or Facebook, and I don't disclose my political opinions. Because I am not a member of the group, I don't have to represent a certain group, and I don't need to promote it. Some of my opinions don't make a big difference even if they are said, so it's okay to talk privately, and it's boring to be in the public. "(Male, 45 years old, assistant professional)

It can be seen that Hong Kong youth were less willing to disclose their personal opinions when it comes to publicly publishing information and speech, especially in the political arena. Higher political media literacy makes them more focused on protecting their information privacy, and it is more cautious to make public statements.

\subsection{Hong Kong youths were not willing to think about national political affairs}

The frequent political incidents have a certain impact on the harmony and stability of Hong Kong society. Will Hong Kong youths spend more time thinking about national political issues? According to the survey results, $47.9 \%$ of respondents did not spend too much time thinking about political issues; $40.1 \%$ of respondents were concerned about political events generally; only $12.0 \%$ of respondents indicated willing to spend time paying attention 
to national events. The one-sample $\mathrm{T}$ test $(\mathrm{t}=-\mathrm{n}$ national political affairs, and the average value 18.878, $\mathrm{P}<0.001$ ) shows that the Hong Kong of this option is 2.51 . youth interviewed were not willing to think about

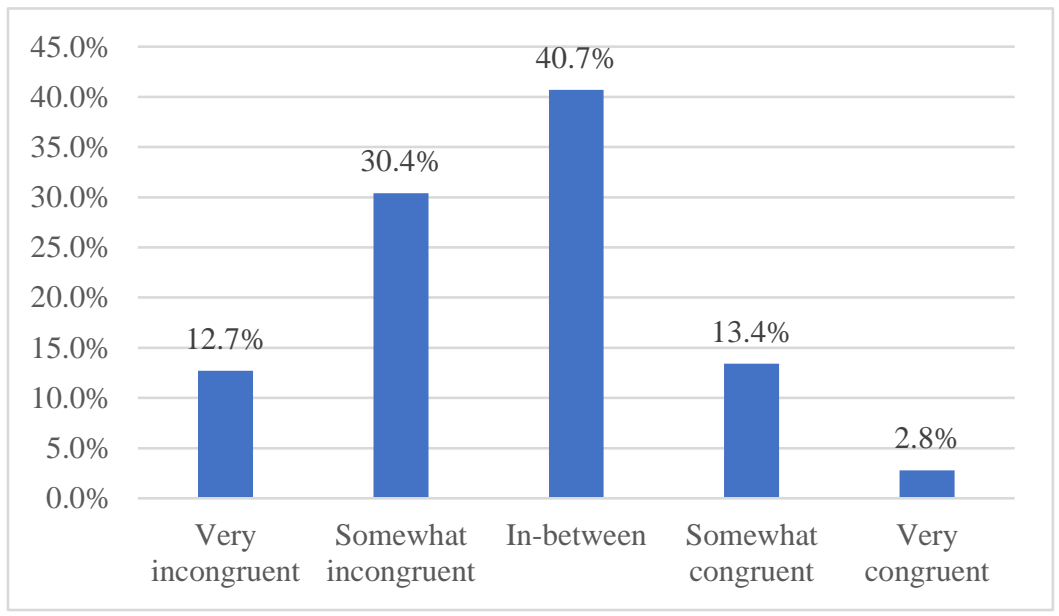

Figure 6: Willingness to express political claims $(\mathrm{N}=1187)$

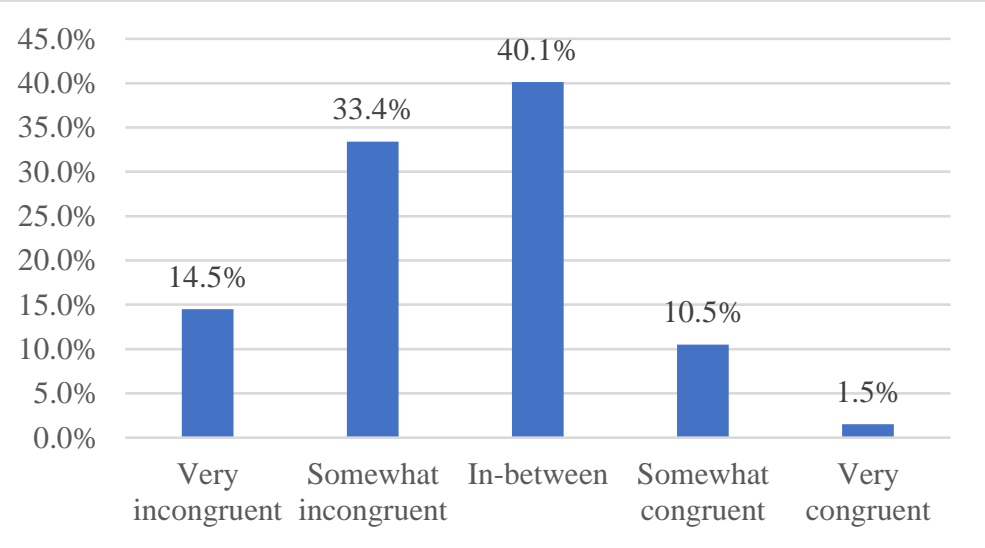

Figure 7: "I am willing to spend a lot of time thinking about national affairs" $(\mathrm{N}=1196)$

5.4 The influence of online media on Hong Kong youths political participation was not significant

Although Hong Kong's online media is well developed and news reports will affect the political attitudes of Hong Kong youths, it is not easy to extend this influence to the actual political participation activities offline. Nearly half of the respondents believed that the online views did not affect people's participation in political action. The proportion of neutral attitude was $32.2 \%$, and only $22.3 \%$ of respondents believed that some people had participated in demonstrations because of network incitement. In general, the influence of online media on Hong Kong youth political participation was not significant.

From the above analysis, it can be seen that Hong Kong was a highly commercial city. Hong Kong people generally lacked interest in political issues. Hong Kong people were mainly concerned with economic issues. They were not keen on participating in political discussion on social media such as Facebook/Twitter, and they Http://escipub.com/international-journal-of-social-research/ 
only choose a limited political expression when interests. something that seriously affects their own

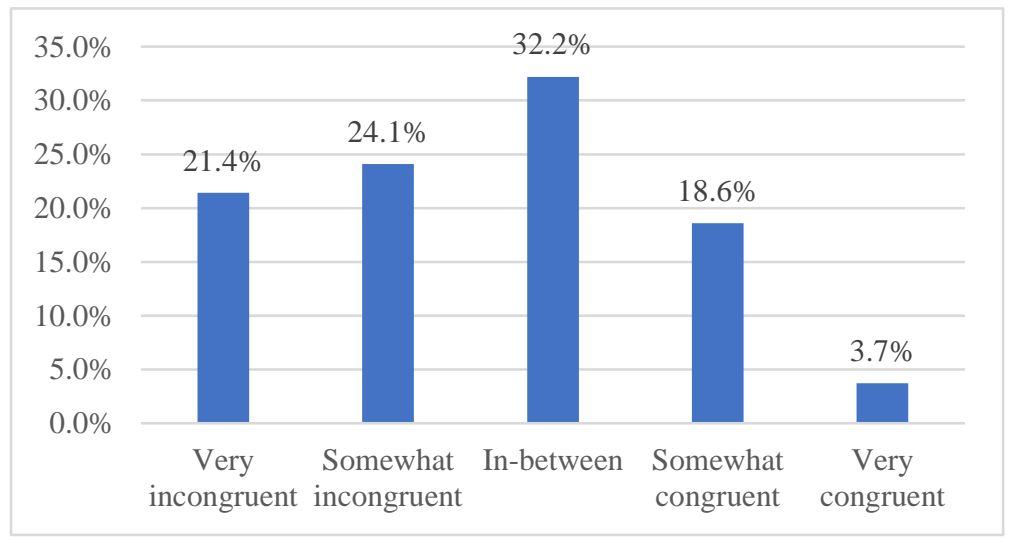

Figure 8: "I have seen people participate in demonstrations due to the influence of the network." ( $N=1193)$

\section{Conclusions}

In recent years, there have been many political incidents in Hong Kong and the media environment is complicated. How to correctly guide the youths to strengthen patriotism and love Hong Kong, and guide them to reasonably express political opinions need to draw a special attention.

This study found that from the perspective of identity, Hong Kong youths had a clear sense of locality. Most people thought that they are "Hong Kong people", followed by "Hong Kong people in China." They were satisfied with their current lives and were relatively optimistic about Hong Kong's future development. Further, they were positive about their future development. A good living environment has made Hong Kong youths show a relatively indifferent tendency in media political literacy. Their interests in political issues were generally low, and they did not spend time thinking about national political events. They would not actively join in the discussion of political topics. In the eyes of Hong Kong youths, media political information would not have much impact on their political participation.
In short, Hong Kong's unique political and economic system, the media environment and many other factors have contributed to the unique identity concept and media political literacy of Hong Kong youths.

\section{References}

1. Deaux, K. (1993). Reconstructing social identity. Personality and Social Psychology Bulletin, 19(1), 4-12.

2. Fu, X., \& Zhang, D. (2016). ransformation of identity: an analysis based on media users' public welfare practice of micro-blog. Modern Communication, 38(10), 48-52.

3. Hall, S., \& Gay, P. d. (2010). Questions of Cultural Identity: Henan University Press.

4. Luo, G., \& Liu, X. (2000). Cultural research Reader: China Social Sciences Press.

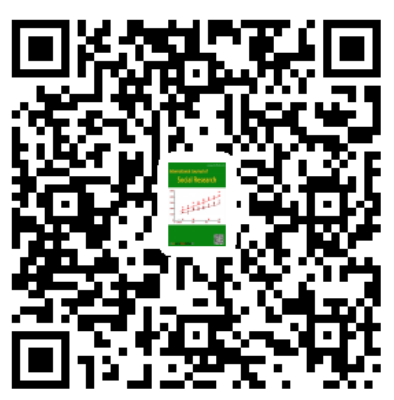

